\title{
David Mitchell, Diversities in Education: Effective Ways to Reach All Learners, Routledge: Abingdon, New York, 2016; 336 pp.: ISBN 9781138924703
}

Reviewed by NIKA ŠUŠTERIČ ${ }^{1}$

One of the central debates in education, at least since the middle of the $20^{\text {th }}$ century, concerns the question of the reproduction of social inequalities. Education itself is supposed to actively contribute to reducing them. It does so primarily by reducing the inequalities in students' achievements, especially if these are produced by contingent attributes or circumstances, such as gender, socioeconomic status, ethnicity and race for example. However, the intensification of individualism, witnessed in the past few decades, shifted the focus to the

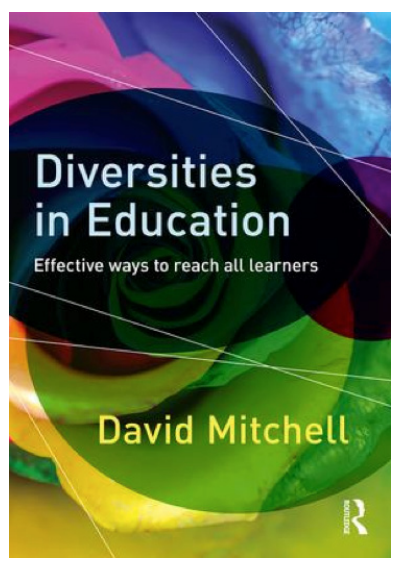
uniqueness of individuals. The concepts of gender, social class, or race seem to have become too narrow to be able to describe the experiences of individuals in schools and beyond. Today, when speaking of social inequalities and the role education has in eliminating them, one comes across another term more and more often: diversity. Diversity is presented as a key characteristic of (mostly Western) societies in the $21^{\text {st }}$ century, with its' recognition as the basis for building community and society. Recognising diversity in education is supposed to enable forming a just society while also acknowledging the entire complexity of every individual and his or her identity. Diversity in education is also the main focus of the book Diversities in Education: Effective Ways to Reach All Learners, written by David Mitchell, an author most known for his work in the fields of special and inclusive education.

Mitchell's book is comprised of six major chapters. The first, titled Samenesses and differences: an introduction, seems to promise an analysis of some crucial terms: sameness, difference, and diversity, to mention the most obvious. Mitchell does offer brief dictionary definitions of diversity and difference, terms he often uses interchangeably, but the chapter mostly comes down to an exposition of Mitchell's views on education, its' tasks and goals, and an explanation of

1 University of Ljubljana, Faculty of Education, Slovenia; susteric.nika@gmail.com. 
certain presumptions that should form their basis.

The other five chapters concern 'the big five', as Mitchell calls them ( $\mathrm{p}$. 2), areas of difference: sex/gender, socioeconomic status/social class, ethnicity/race/culture, religious beliefs and abilities. These five chapters are mostly similar in their structure - the introductory definitions are followed by analyses of the effects certain differences have on the educational achievements of students. These are followed by propositions for reducing inequalities in education, arising from the aforementioned differences. In accordance with his ecological perspective (p. 15-16), Mitchell proposes solutions on systemic, school and classroom levels (the chapter on religious beliefs is an exception to which we will return later).

The second chapter, Sex and gender differences, starts by defining the terms sex and gender. What follows is an eclectic collection of differences between (the two) genders/sexes. Probably the greatest shortcoming of the chapter lies in the approach the author uses for explaining these differences. He separately lists a few possible reasons for differences that relate either to nature or to nurture. Despite his inclination towards an interactive approach to the topic, he does not offer an analysis that would combine both strands and thus fails to adequately shed light on the ways in which, for example, certain biological differences gain moral value which in turn significantly shape individual biographies and trajectories.

Whereas the chapter on gender/sex mostly focuses on differences between genders in general, the third chapter, Social class/socio-economic status differences, centres more on differences in educational achievement that arise from differences in students' social backgrounds. Mitchell stresses the effects of various factors ranging from differences in cultural and social capital to differences in students' diets. Among the many propositions for eliminating these inequalities, Mitchell also stresses the importance of appropriate funding models for education and so moves away from current trends in education policy that attempt to shift the responsibility for reducing inequality to individual schools and teachers.

The fourth chapter, Race/ethnicity/culture differences, follows the same structure as the two chapters preceding it. Mitchell directly acknowledges that countries differ significantly in their ethnic compositions and histories (p. 152), which makes it difficult to propose simple solutions for reducing inequalities caused by ethnic differences. Mitchell frequently points out that the discussion of the effects ethnicity has on educational achievement must consider the interactions between various factors involved in forming individuals, including at least gender and socio-economic status. 
The next chapter, Religions/beliefs differences, is distinct from all the other chapters. Mitchell abandons the structure used in previous chapters and does not discuss the question of differences in educational outcomes arising from students' religious beliefs or practices. He does start the chapter by briefly discussing what religion and its' place in society is, but what follows concerns the role of religious and religion education in schools. Mitchell offers an overview of certain national and international conventions concerning the rights regarding religious beliefs and follows up with a comparison of different national models of religious and religion education. While he expresses his opinion against religious indoctrination in education clearly, he unfortunately does not analyse inequalities arising from students' religious beliefs. The issue is certainly complex and demanding, but this only gives all the more reason for conceptualising it and finding mechanisms to reduce educational inequalities based on religion.

The fifth chapter is titled Different abilities. Mitchell offers an overview of the most prominent approaches in educating children with different abilities, or, to put it differently, of children with special educational needs. He explains what some of the reasons for different abilities are and continues with a rather elaborate description of propositions for creating an education accessible to all students. The author, mostly known for his work in the area of special and inclusive education, does not hide his affinity for inclusive education as an appropriate model for education in general. Throughout the book, Mitchell expresses the ever more popular view that inclusion is not concerned with educating children with special needs only, but presents a model of education that can, in combination with personalisation and the universal design for learning, cater to the needs of each and everyone comprising a diverse population of students.

To conclude our review, we should emphasise that the issues discussed in Diversities in Education are complex and extensive and perhaps too demanding to be adequately discussed within a single book. This is also probably why the analyses of differences and their causes offered by Mitchell are often fragmented. While Diversities in Education can present a possible entry point to acquainting oneself with issues of equity in contemporary education it also, perhaps even more importantly, invites us to think about the concept of diversity itself. It seems, after all, that we are dealing with a concept that promises us to quite simply solve one of the fundamental tensions in education: that between the individual and the collective. However, reducing persistent and, to a degree, immanent tensions with a 'one concept solves all' approach might cause diversity to fall short of expectations. 